\title{
MAIT cells in Type 1 Diabetes: a good friend turned bad
}

Lina Petersone and Lucy S.K. Walker

Institute of Immunity \& Transplantation, University College London Division of Infection \& Immunity, Royal Free Campus, London, UK NW3 2PF

Corresponding author: Prof Lucy S.K. Walker, Institute of Immunity \& Transplantation, University College London Division of Infection \& Immunity, Royal Free Campus, London, UK NW3 2PF. Tel: +44 (0)20 77940500 ext 22468. Email: lucy.walker@ucl.ac.uk 
Mucosal-associated invariant T (MAIT) cells normally preserve gut barrier integrity, but can switch their phenotype to play a pathogenic role in type 1 diabetes

The iterative dialogue between gut microbiota and the immune system has emerged as a dominant theme in immunology research. But how microbial cues shape the development of immune-mediated disease remains unclear. In this issue of Nature Immunology, Rouxel et al. suggest that mucosal-associated invariant $T$ (MAIT) cells responding to microbial metabolites can influence the development of type 1 diabetes ${ }^{1}$.

Type 1 diabetes (T1D) is a complex autoimmune disease that leads to destruction of insulinproducing pancreatic $\beta$-cells and a requirement for lifelong exogenous insulin administration ${ }^{2}$. While the pathophysiology of T1D is still not entirely understood, a growing body of evidence implicates alterations in gut permeability and changes to the intestinal microbiota as early events in disease development ${ }^{3,4}$. MAIT cells are innate-like T cells that are enriched in the intestine and express a T cell receptor (TCR) composed of an invariant TCR $\alpha$ chain (TRAV1-2-TRAJ33/12/20 in humans and TRAV1-TRAJ33 in mice) coupled with a limited repertoire of TCR $\beta$ chains. This endows them with the capacity to recognise bacteriaderived riboflavin precursors presented by the major-histocompatibility-complex-class-Irelated protein 1 (MR1) $)^{5}$ MAIT cell development is impaired in germ-free mice ${ }^{6}$, emphasizing their intimate connection with commensal microflora.

To probe the possible involvement of MAIT cells in T1D, Rouxel et al. first assessed their number in the peripheral blood of children who had developed T1D within the last 10 days. MAIT cell frequencies were significantly reduced in these children compared with agematched controls and their phenotype was also distinct, with higher CD25 and PD-1 and lower CCR6 and CD56 than in control children. In addition, PMA-ionomycin stimulated MAIT 
cells from children with recent onset T1D produced less IFN- $\gamma$ but more TNF, IL-4 and Granzyme B. Remarkably, measurement of just 4 markers (CCR6, CD25, PD-1, CD56) on MAIT cells in the blood was sufficient to define a predictive diagnostic model capable of distinguishing the newly diabetic children from healthy controls.

Curiously, the authors identified a relationship between MAIT cell Granzyme B expression and age of T1D diagnosis, with a young age at diagnosis being associated with high frequencies of Granzyme $\mathrm{B}^{+}$MAIT cells. This is interesting in the light of the growing recognition that children diagnosed young appear to show a more aggressive disease course and have fewer insulin-expressing pancreatic islets at diagnosis ${ }^{7}$. Spurred on by this finding, the authors began to wonder whether MAIT cells could use their cytotoxic potential to directly contribute to pancreatic $\beta$-cell death. Staging the perfect crime scene, they cocultured purified MAIT cells with the human $\beta$-cell line EndoC- $\beta \mathrm{H} 1$, throwing in the MAIT cell ligand 5-OP-RU to initiate interactions. However, the anticipated killing spree never materialised. Mindful that cell surface MR1 levels are low on most cell types ${ }^{8}$, the authors added inflammatory cytokines to the mix, opting for IL-1 $\beta$, IFN- $\gamma$, and TNF since these are typically present in inflamed islets in the T1D setting. Strikingly, MR1 expression rose on the EndoC- $\beta \mathrm{H} 1$ cells and the MAIT cells became activated and degranulated, eliciting killing that was dependent on ligand dose and blocked by anti-MR1 antibodies. Future work is needed to assess whether MAIT cells are present at the scene of the crime in the pancreas of diabetic humans, and whether primary $\beta$-beta cells can indeed express MR1 in situ. Nevertheless, these provocative findings suggest the potential for MAIT cells to directly participate in the process of $\beta$-cell destruction (Figure 1). 
In mice, MAIT cells comprise only $\sim 0.1 \%$ of peripheral blood T cells compared to $\sim 6 \%$ in humans ${ }^{9}$, and until recently the study of murine MAIT cells was limited by a lack of sensitive identification methods. However, the development of riboflavin derivative 5-OP-RU-loaded MR1 tetramers has allowed researchers to recapitulate in mouse models many of the observations previously made in human MAIT cells ${ }^{9}$. Taking advantage of this research tool, Rouxel et al. turned to the NOD mouse model of T1D. In general the frequencies of MAIT cells were lower in NOD than in the C57BL/6 mice that were used as a control, and were even below detection levels in the blood. Interestingly however MAIT cells were present in the pancreatic islets of NOD mice and were more abundant in the ileum of NOD than C57BL/6 animals.

One limitation of human studies is the understandable reliance on peripheral blood samples and the inability to simultaneously explore multiple tissue sites. Taking advantage of the mouse model, Rouxel et al. were able to gain a more comprehensive view of how MAIT cells redistribute during disease development. Interestingly, progression to diabetes in NOD mice was associated with a trend towards lower MAIT cell frequencies in the ileum and spleen and a concomitant increase in the pancreatic lymph nodes and pancreatic islets. Adoptive transfer experiments confirmed that MAIT cells could directly migrate from the blood to the tissues, with a strong preference for the ileum over the islets in young NOD mice, but a shift towards islet migration in older animals.

Most interesting was the change in MAIT cell phenotype during disease progression. In 16wk old mice, islet-resident MAIT cells already expressed some Granzyme B, but in diabetic animals levels were much higher and IFN- $\gamma$ was also produced. Conversely, in the ileum MAIT cells produced IL-22 and IL-17 in 16wk old mice but this was virtually extinguished in 
diabetic animals. These kinetic data capture the shift in MAIT cell behaviour as they forsake their role in gut homeostasis to join the conventional T cells waging war in the islets. So what is the trigger for this shift? The authors noted a decrease in IL-23 levels in the ileum at diabetes onset which likely explains the drop in MAIT cell IL-22 and IL-17 production. Intriguingly this coincided with an increase in gut permeability, as measured by blood FITCdextran levels after oral gavage, raising the possibility that MAIT cell alterations are instructed by microbe-derived ligands.

To directly test the role of MAIT cells in diabetes pathogenesis, the authors painstakingly generated $\mathrm{MrI}^{-/-} \mathrm{NOD}$ mice and compared them with co-housed littermate $\mathrm{MrI}^{+/-}$controls to avoid differences in gut microbiota. The lack of MAIT cells in the absence of MR1 led to severely damaged gut epithelium, increased intestinal permeability and greater immune activation resulting in significantly exacerbated disease. These data reveal a protective role for MAIT cells in T1D development centering on their role in maintaining gut integrity. They also indicate (unsurprisingly) that MAIT cells are not absolutely required for islet destruction, or for sensing the loss of gut barrier integrity, even though they may contribute to these processes.

One question arising from these studies echoes the classic chicken and egg conundrum: Does impaired MAIT cell function trigger loss of gut integrity, or does a leaky gut stimulate MAIT cell changes? Given the smorgasbord of susceptibility loci that influence real world autoimmunity, it is easy to envisage that both scenarios might exist, with some individuals harbouring single nucleotide polymorphisms that alter MAIT cell activation and/or homing and others being genetically predisposed to increased gut permeability. A deeper 
understanding of MAIT cell biology could potentially shed light on the association between T1D and celiac disease, given the link to gut permeability in both conditions.

Arguably the most significant finding of the study was that MAIT cell changes could be detected in humans even before the development of diabetes. Accordingly, relatives of people with T1D with at least 2 autoantibodies had blood MAIT cells showing similar phenotypic alterations to those seen in the new onset patients. Since risk profiles differ between relatives with a single autoantibody versus multiple autoantibodies, it would be of great interest to compare MAIT cell phenotypes between these groups and assess the relationship with disease progression. Perhaps tracking MAIT cells can help discern which individuals in the "at risk" category will go on to develop disease.

More generally, the findings add to a growing body of work highlighting environmental influences on autoimmune disease development. Over the past decades, the incidence of T1D has increased dramatically at a rate that suggests involvement of factors beyond genetic change. At the same time, it has become apparent that the immune system is shaped to a surprising extent by non-heritable influences ${ }^{10}$. The microbiota undoubtedly exerts a major influence on immunity, with recent suggestions that bacteria can even translocate from the gut to the pancreatic lymph nodes in a mouse model of diabetes ${ }^{11}$. The new findings from Rouxel et al. position MAIT cells at the nexus between microbiota and immune function, limiting commensal dissemination by preserving gut integrity and in turn being stimulated by microbial products. Tantalisingly, although MAIT cells recognise bacterial metabolites, recent evidence suggests they are also activated during viral infections $^{12}$. Given the putative links between viral infection and T1D, this promises to be an active area of future investigation. 


\section{References}

1. Rouxel, O. et al. Nat. Immunol.

2. Atkinson, M. A., Eisenbarth, G. S. \& Michels, A. W. Lancet 383, 69-82 (2014).

3. Vaarala, O., Atkinson, M. A. \& Neu, J. Diabetes 57, 2555-2562 (2008).

4. Paun, A., Yau, C. \& Danska, J. S. J. Immunol. 198, 590-595 (2017).

5. Kjer-Nielsen, L. et al. Nature 491, 717-723 (2012).

6. Treiner, E. et al. Nature 422, 164-169 (2003).

7. Leete, P. et al. Diabetes 65, 1362-1369 (2016).

8. Franciszkiewicz, K. et al. Immunol. Rev. 272, 120-138 (2016).

9. Rahimpour, A. et al. J. Exp. Med. 212, 1095-1108 (2015).

10. Brodin, P. et al. Cell 160, 37-47 (2015).

11. Costa, F. R. C. et al. J. Exp. Med. 213, 1223-1239 (2016).

12. van Wilgenburg, B. et al. Nat. Commun. 7, 11653 (2016). 
Protective

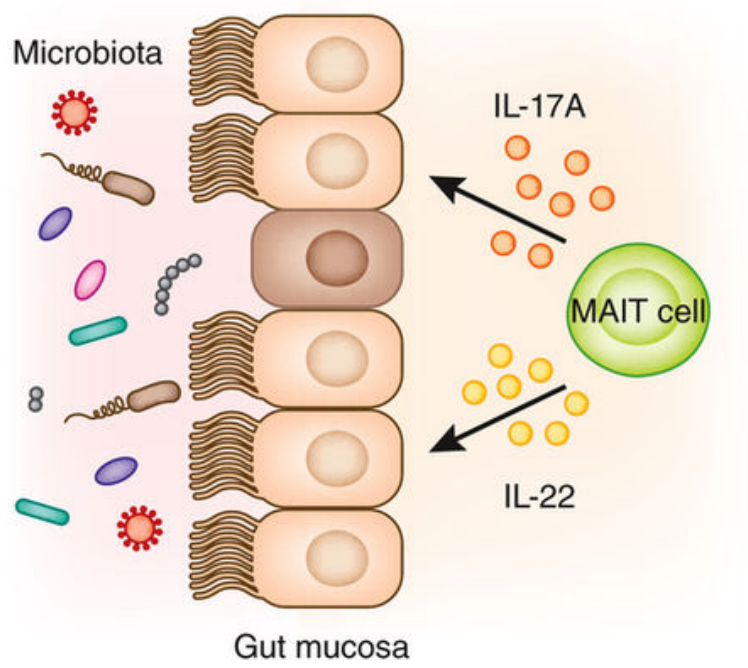

Pathogenic

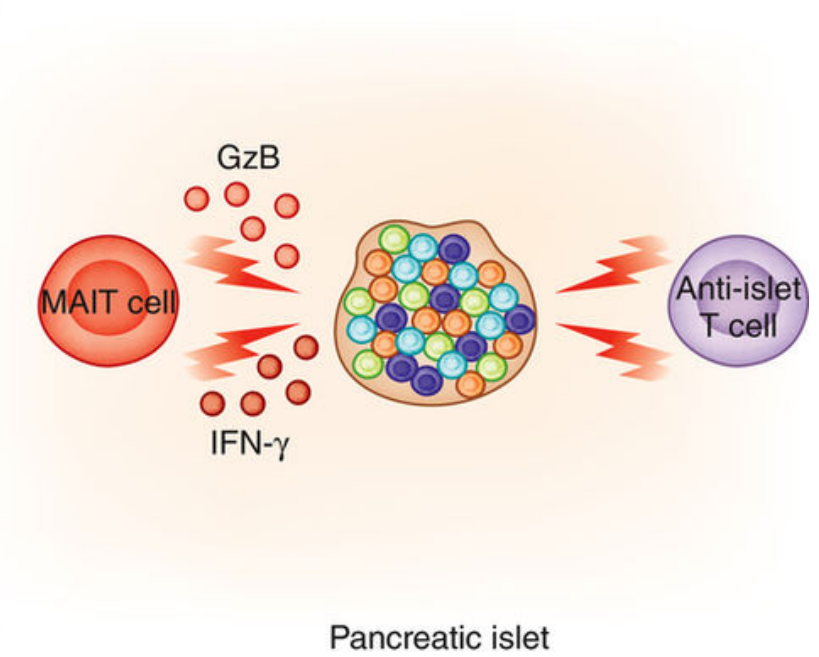

Figure 1: Dual role of MAIT cells in T1D. MAIT cells are enriched in the gut and recognize bacterial metabolites. They produce IL-17A and IL-22 which are important for the maintenance of gut barrier integrity. Changes in gut permeability and intestinal microbiota have been linked to T1D development in humans. NOD mice lacking MAIT cells show increased gut permeability and exacerbated disease, suggesting MAIT cells can protect from T1D. In NOD mice, progression to diabetes is associated with decreased production of IL17A and IL-22 from MAIT cells in the ileum and an accumulation of IFN- $\gamma$ and Granzyme B producing MAIT cells in the pancreatic islets. Human MAIT cells can directly kill a human $\beta$ cell line suggesting a potential pathogenic role in T1D.

\section{Competing Financial Interests}

The authors declare no competing financial interests 